cells could lead to an autoimmune component in HTLV-I infection. For example, Sjögren syndrome, which is associated with HAM/TSP, might be a result of unregulated expansion of autoreactive $\mathrm{CD} 4^{+} \mathrm{T}$ cells leading to increased numbers of autoantibodies as well as lymphoproliferation and systemic inflammatory changes and the presence of infiltrating T cells. Viruses have evolved to subvert the host cells they infect, leading to unexpected consequences. The findings by Yamato et al. could help explain why certain viral infections are often associated with autoimmune diseases through their inhibition of cellular luxury function.

Address correspondence to: Robert S. Fujinami, Department of Neurology, University of Utah School of Medicine, 30 North 1900 East, 3R330 School of Medicine, Salt Lake City, Utah 84132, USA. Phone: (801) 5853305; Fax: (801) 585-3311; E-mail: Robert. Fujinami@hsc.utah.edu.

1. Uchiyama, T. 1997. Human T cell leukemia virus type I (HTLV-I) and human diseases. Annu. Rev. Immunol. 15:15-37.

2. Yamano, Y., et al. 2005. Virus-induced dysfunction of CD $4^{+} \mathrm{CD} 25^{+} \mathrm{T}$ cells in patients with HTLV-I-associated neuroimmunological disease. J. Clin. Invest. 115:1361-1368. doi:10.1172/JCI200523913.

3. Oldstone, M.B.A., et al. 1982. Virus-induced alterations in homeostasis: alteration in differentiated functions of infected cells in vivo. Science. 218:1125-1127.

4. Orland, J.R., et al. 2003. Prevalence and clinical features of HTLV neurologic disease in the HTLV Outcomes Study. Neurology. 61:1588-1594.

5. Murphy, E.L., et al. 1997. Increased prevalence of infectious diseases and other adverse outcomes in human T lymphotropic virus types I- and II-infected blood donors. Retrovirus Epidemiology Donor Study (REDS) Study Group. J. Infect. Dis. 176:1468-1475.

6. Okayama, A., et al. 1997. Increased expression of interleukin- 2 receptor $\alpha$ on peripheral blood mononuclear cells in HTLV-I tax/rex mRNA-positive asymptomatic carriers. J. Acquir. Immune Defic. Syndr. Hum. Retrovirol. 15:70-75.

7. Satoh, M., et al. 2002. Involvement of IL-2/IL-2R system activation by parasite antigen in polyclonal expansion of CD4 ${ }^{+} 25^{+}$HTLV-1-infected T-cells in human carriers of both HTLV-1 and S. stercoralis. Oncogene. 21:2466-2475.

8. Yamano, Y., et al. 2004. Increased expression of human T lymphocyte virus type I (HTLV-I) Tax11-19 peptide-human histocompatibility leukocyte antigen $\mathrm{A}^{*} 201$ complexes on $\mathrm{CD}^{+} \mathrm{CD}^{2} 5^{+} \mathrm{T}$ cells detected by peptide-specific, major histocompatibility complexrestricted antibodies in patients with HTLV-I-associated neurologic disease. J. Exp. Med. 199:1367-1377.

9. Piccirillo, C.A., and Shevach, E.M. 2001. Cutting edge: control of $\mathrm{CD}^{+} \mathrm{T}$ cell activation by $\mathrm{CD} 4^{+} \mathrm{CD} 25^{+}$ immunoregulatory cells. J. Immunol. 167:1137-1140.
10. Yoshida, M., et al. 1989. Increased replication of HTLV-I in HTLV-I-associated myelopathy. Ann. Neurol. 26:331-335

11. Ono, A., Mochizuki, M., Yamaguchi, K., Miyata, N., and Watanabe, T. 1995. Increased number of circulating HTLV-1 infected cells in peripheral blood mononuclear cells of HTLV-1 uveitis patients: a quantitative polymerase chain reaction study. $\mathrm{Br}$. J. Ophthalmol. 79:270-276.

12. Greenberg, S.J., Jacobson, S., Waldmann, T.A., and McFarlin, D.E. 1989. Molecular analysis of HTLV-I proviral integration and $\mathrm{T}$ cell receptor arrangement indicates that $\mathrm{T}$ cells in tropical spastic paraparesis are polyclonal. J. Infect. Dis. 159:741-744.

13. Gessain, A., et al. 1985. Antibodies to human T-lymphotropic virus type-I in patients with tropical spastic paraparesis. Lancet. 2:407-410.

14. Nakagawa, M., et al. 1995. HTLV-I-associated myelopathy: analysis of 213 patients based on clinical features and laboratory findings. J. Neurovirol. 1:50-61.

15. Fehérvari, Z., and Sakaguchi, S. 2004. CD4 ${ }^{+}$Tregs and immune control. J. Clin. Invest. 114:1209-1217. doi:10.1172/JCI200423395.

16. Fehérvari, Z., and Sakaguchi, S. 2004. Development and function of $\mathrm{CD} 25^{+} \mathrm{CD} 4^{+}$regulatory T cells. Curr. Opin. Immunol. 16:203-208.

17. Walker, M.R, et al. 2003. Induction of FoxP3 and acquisition of $\mathrm{T}$ regulatory activity by stimulated human CD4 ${ }^{+} \mathrm{CD} 25^{-} \mathrm{T}$ cells. J. Clin. Invest. 112:1437-1443. doi:10.1172/JCI200319441.

18. Jacobson, S., Shida, H., McFarlin, D.E., Fauci, A.S. and Koenig, S. 1990. Circulating $\mathrm{CD}^{+}$cytotoxic $\mathrm{T}$ lymphocytes specific for HTLV-I pX in patients with HTLV-I associated neurological disease. Nature. 348:245-248

\title{
A seek-and-hide game between Cd1-restricted T cells and herpesviruses
}

\author{
Nagendra R. Hegde and David C. Johnson
}

\author{
Department of Molecular Microbiology and Immunology, Oregon Health and Science University, Portland, Oregon, USA.
}

\begin{abstract}
T and NK cells collaborate to control viral infections, discerning minute differences between infected and uninfected cells. At the same time, viruses have evolved to escape this discovery. In this issue of the JCI, Ganem and colleagues show that Kaposi sarcoma-associated herpesvirus (KSHV) inhibits CD1d presentation to $T$ cells (see the related article beginning on page 1369). This novel immune evasion strategy highlights the importance of CD1d-restricted $\mathrm{T}$ cells in controlling viral infection and raises an interesting question: how do $T$ cells recognize viruses in the context of CD1 molecules that bind lipids? In the case of herpesviruses, alterations in endosomal trafficking might trigger redistribution of CD1/lipid complexes to cell surfaces, thereby promoting recognition by CD1d-restricted $\mathrm{T}$ cells.
\end{abstract}

Nonstandard abbreviations used: HCMV, human cytomegalovirus; HSV, herpes simplex virus; KSHV, Kaposi sarcoma-associated herpesvirus; MIC, MHC class I chain-related; MIR, modulator of immune recognition; TGN, trans-Golgi network; ULBP, UL16-binding protein; US2, unique short region open reading frame 2.

Conflict of interest: The authors have declared that no conflict of interest exists.

Citation for this article: J. Clin. Invest. 115:1146-1149 (2005). doi:10.1172/JCI200525000.

\section{Recognition of viruses}

Control of viruses by the immune system is critically dependent upon recognizing differences between infected and uninfected cells. It is also imperative that immune cells identify viruses inside cells before progeny are produced and can spread. Early recognition relies on detecting the appearance of new cell-surface markers or the loss of oth- ers. The classic examples are MHC class I and MHC class II molecules, which present peptides derived from viral proteins to $T$ lymphocytes, which use variable TCRs to discern the viral peptides against a huge background of cellular peptides. NK cells recognize cells that lack cell-surface MHC class I molecules, a situation caused by viral inhibition of the class I pathway. Viral infection can also trigger the appearance of new molecules detectable by NK cells. For example, genes encoding MHC class I chain-related-A (MIC-A) and MIC-B proteins and UL16-binding protein (ULBP) are coupled to hair-trigger promoters that are "tripped" by viruses so that cell-surface MIC proteins and ULBPs are detected by NK group 2D (NKG2D) receptors on NK and $\mathrm{CD} 8^{+} \mathrm{T}$ cells (1).

\section{Viral immune evasion}

As the immune system endeavors to recognize the presence of a virus inside cells, 
Table 1

Viral proteins affect multiple immune signals

\begin{tabular}{|c|c|c|}
\hline Virus & Viral immune modulator & Host immune recognition molecules affected \\
\hline \multirow[t]{4}{*}{ HCMV } & US2 & $\begin{array}{l}\text { MHC class I HC (HLA-A, -B, -C, - -G), } \\
\text { MHC class Il } \alpha, \text { HLA-DM } \alpha, \text { HFE, MR1 }\end{array}$ \\
\hline & US3 & MHC class I, MHC class II \\
\hline & US11 & MHC class I HC, MR1 \\
\hline & UL16 & MIC-B, ULBP-1, ULBP-2 \\
\hline & Nef & MHC class I, MHC class II, CD4, CD28, CD1a \\
\hline \multirow{2}{*}{ KSHV } & MIR1 (K3) & MHC class I (HLA-A, -B, -C, -E), CD1d \\
\hline & MIR2 (K5) & $\begin{array}{c}\text { MHC class I (HLA-A, -B, -C, -E), } \\
\text { B7.2, ICAM-1, CD1d }\end{array}$ \\
\hline Myxomavirus & M153R & MHC class I, CD4, Fas \\
\hline
\end{tabular}

HFE, hemochromatosis (loss-of-function mutations in which cause hereditary hemochromatosis); $\mathrm{K} 3$, KSHV open reading frame 3; MHC class I HC, MHC class I heavy chain; MR1, MHC class Irelated; UL, unique long.

the viruses have evolved to modulate this response. Viruses obstruct most or all steps of the MHC class I and MHC class II antigen-presentation pathways (2-4). Viral proteins prevent transport of antigenic peptides from the cytosol into the $\mathrm{ER}$, induce degradation of MHC class I and MHC class II proteins, and mislocalize or divert these cellular proteins from their normal trafficking pathways. Herpesviruses also replace host MHC class I proteins with viral homologs and prevent cell-surface expression of MIC-B and ULBPs in order to fool NK cells scanning for lack of MHC class I expression $(3,5)$.

\section{CD1, CD1-restricted T cells, and viruses}

CD1 proteins are MHC class I molecules that present lipids and glycolipids to $T$ cells through their TCRs $(6,7)$. Recognition of $\mathrm{CD} 1$ in the context of viral infection is not well understood. Human CD1 molecules are encoded by 5 genes, $C D 1 A, C D 1 B, C D 1 C$, $C D 1 D$, and $C D 1 E$, and each protein has different lipid-binding specificities and trafficking properties. CD1d-restricted $\mathrm{T}$ cells include NK T cells that express CD161 (corresponding to NK1.1) and frequently possess invariant TCRs as well as $T$ cells with diversely rearranged TCRs $(6,7)$. It is well established that CD1- and CD1d-restricted $\mathrm{T}$ cells play essential or important roles in controlling bacterial infections, especially in the case of infection with mycobacteria (6-8). Control of such infection can be readily understood, as bacteria express novel lipid antigens. Bacterial glycolipids are largely taken up into endosomes and lysosomes before being loaded onto CD1 (6). A lipid derived from a marine sponge, $\alpha$-galactosylceramide ( $\alpha$-GalCer), is also a potent antigen for CD1d-restricted $T$ cells and has been used to stimulate both antibacterial and antiviral immunity (9). However, there is substantial evidence that lipids derived from host cell membranes can stimulate CD1-restricted T cells (6). Frequently activated in early phases of the immune response, CD1-restricted $\mathrm{T}$ cells stimulate NK, T, and B cells, macrophages, and DCs through IFN- $\gamma$, IL-2, and IL-4. These $T$ cells are also potently cytolytic and able to release perforin and granzymes and utilize Fas/Fas ligand to kill cells.

CD1- and CD1d-restricted T cells also appear to play important roles in controlling viral infections. $\mathrm{CD} 1 \mathrm{~d}^{-/-}$mice exhibit reduced clearance and increased pathology after infection with respiratory syncytial virus, encephalomyocarditis virus, and coxsackievirus B3 (7-9). CD1-restricted T cells also induce immunopathology or have limited effects on viral infections (10). However, some of the clearest evidence in support of an essential role for CD1d-restricted T cells has come from studies of herpesviruses. Herpes simplex virus-1 (HSV-1) and HSV-2 produce much more profound pathology in $\mathrm{CD} 1 \mathrm{~d}^{-/-}$mice, with markedly increased virus replication and pathology in epithelial tissues, spread to the nervous system, and encephalitis $(11,12)$. Murine cytomegalovirus replication is reduced in mice treated with $\alpha$-GalCer (10). Moreover, a patient lacking NK T cells (and with no other obvious immunodeficiencies) presented with disseminated varicella zoster virus (VZV) after vaccination with attenuated virus (13).

Despite evidence that CD1-restricted T cells are involved in antiviral immunity, it is not yet understood how CD1-restricted
$\mathrm{T}$ cells recognize virus-infected cells. Bacteria express novel lipids, but there is little evidence that viral infections lead to substantial modification of the lipid content of host cells. While many viral proteins are fatty acid modified and glycophosphatidylinositol anchored, these modifications are unlikely to be specific to viruses because cellular proteins are similarly modified. There is evidence that CD1d-restricted T cells are activated without direct recognition of pathogen-derived lipids. This occurs through stimulation by weak selflipid responses presented via CD1d and is then amplified by IL-12 produced by DCs when they detect bacterial products (14). According to this model, recognition of pathogens is achieved by other cells, and CD1-restricted $\mathrm{T}$ cells are indirectly stimulated to express cytokines. However, there are also indications that $\mathrm{CD} 1$-restricted $\mathrm{T}$ cells can recognize virus-infected cells. For example, CD1-restricted $\mathrm{T}$ cells are frequently cytolytic and thus capable of directly interacting with and killing virusinfected cells (6-9).

\section{KSHV inhibition of CD1d-restricted $T$ cell recognition}

In this issue of the JCI, Ganem and colleagues describe loss of cell-surface CD1d in cells infected with KSHV (15). KSHV proteins modulator of immune recognition-1 (MIR1) and MIR2 (also known as K3 and $\mathrm{K} 5$, respectively) caused cell-surface CD1d to be internalized by a process dependent on both the presence of dynamin and the ubiquitination of a CD1d cytoplasmic lysine. Internalized CD1d was relatively stable and was not immediately degraded in lysosomes (15). Previous observations from this laboratory demonstrated that MIR2 also promotes internalization of MHC class I, B7.2, and ICAM-1 molecules $(16,17)$. Not only does MIR2 reduce cell-surface CD1d, but it also inhibits recognition by $\mathrm{CD} 1 \mathrm{~d}$-restricted $\mathrm{T}$ cells. These results are especially interesting because, as the authors point out, they reinforce the notion that $\mathrm{CD} 1 \mathrm{~d}$ in virus-infected cells can signal directly to $T$ cells. Otherwise, why would KSHV infection pull CD1d off the cell surface, a process that can only benefit the virus in infected cells, and not in some other APC that is not infected?

\section{Promiscuity of viral immune evasion proteins}

The results of Sanchez, Gumperz, and Ganem (15) highlight another emerging principle of viral immune evasion. Initial 
A

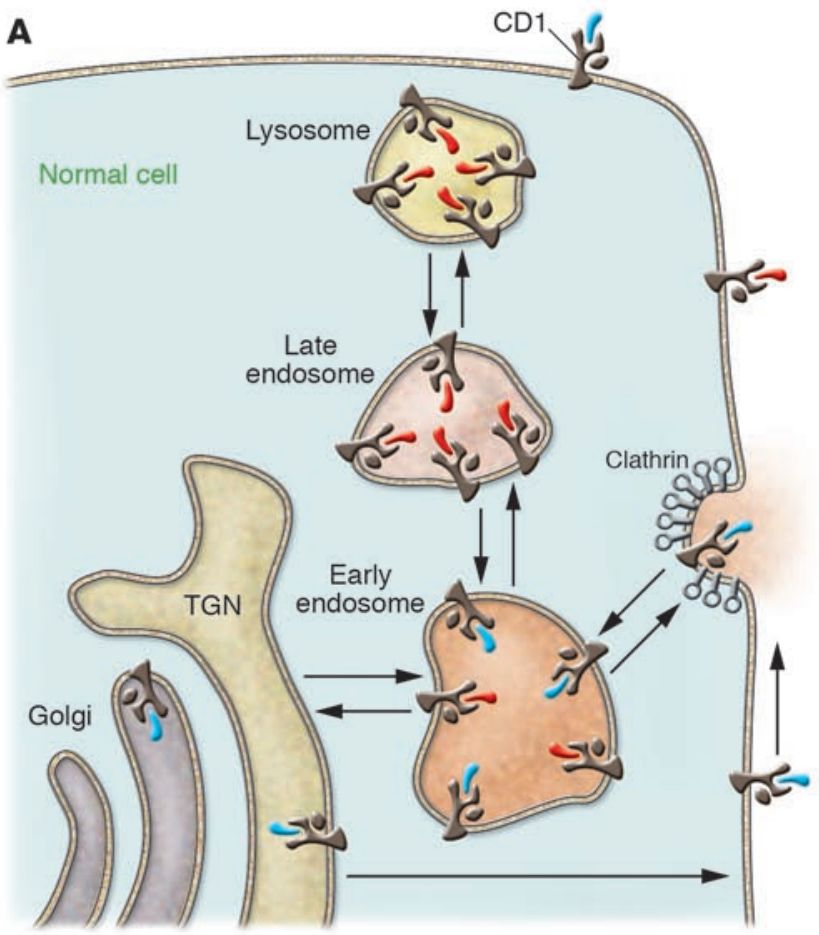

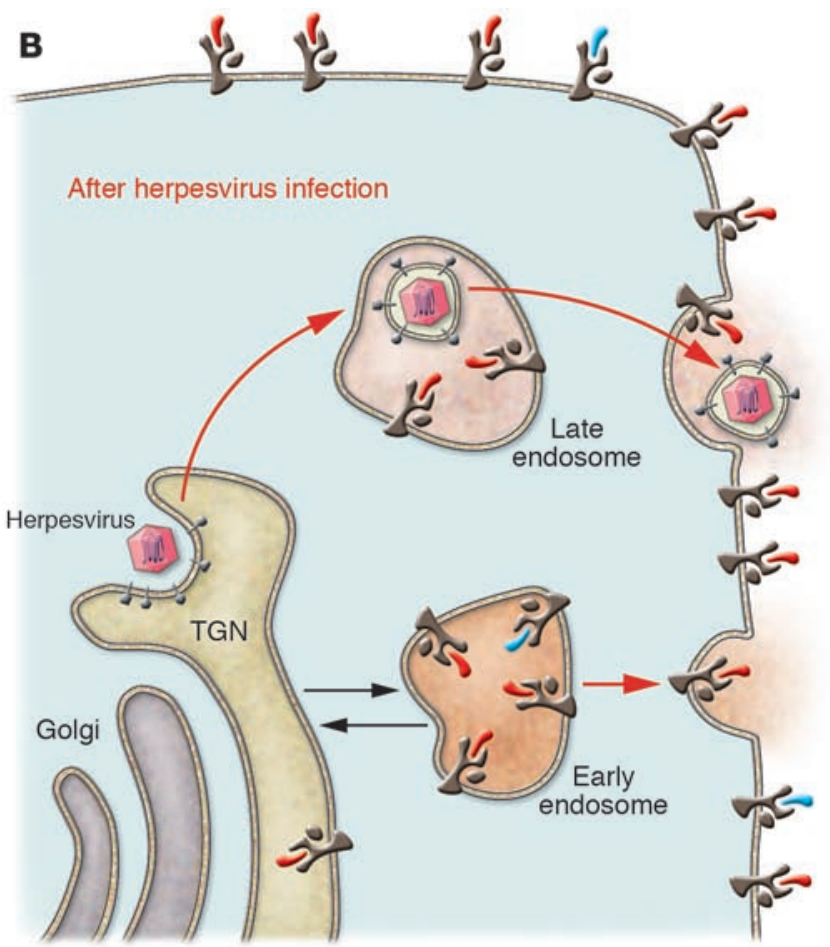

Figure 1

Model for CD1-restricted recognition of herpesvirus infection. (A) Heterodimers of CD1 heavy chain and $\beta_{2} m$ assemble with certain endogenous lipids (blue) in the ER and move through the Golgi apparatus to the cell surface. In normal cells, the majority of CD1 molecules accumulate in endosomes or lysosomes, where there is exchange of ER (or cell-surface) lipids with endosomal lipids (red). Following the acquisition of lipids, very few CD1 molecules return to the plasma membrane. Thus, CD1-restricted T cells that recognize bacterial or self lipids loaded in endosomes and/or lysosomes are not stimulated. Cell-surface CD1d molecules are internalized via clathrin-coated pits. (B) During herpesvirus infection there is assembly of virus particles and budding into TGN and endosomes. Following assembly, viral proteins promote substantial redistribution of TGN/endosomes and their contents to the plasma membrane. Mass movement of endosomes also relocalizes large amounts of CD1 with endosome-derived lipids to the cell surface. This could serve as a recognition signal that host cells are infected with a herpesviruses, causing CD1-restricted T cells to kill the target cell.

reports describing inhibitors of the MHC class I pathway tended to ignore effects of viral proteins on other MHC and nonMHC molecules (2). However, as work has progressed, it has become clear that many viral immune evasion proteins bind multiple cellular targets, thereby preventing recognition by several pathways $(3,4)$. One of the best examples is human cytomegalovirus (HCMV) glycoprotein unique short region open reading frame 2 (US2) that induces degradation of several diverse $\mathrm{MHC}$ class I molecules (HLA-A, HLA-B, HLA-C, HLA-E, and HLA-G), as well as MHC class II $\alpha$ chains (but not $\beta$ or invariant chains), HLA-DM $\alpha$, and the hemochromatosis protein (see Table 1). We recently found that US2 and the closely related protein US11 induce the degradation of the protein product of the MCH complex class I-related (MR1) gene (N.R. Hegde, unpublished observations). KSHV MIR2 reduces recognition by $\mathrm{CD}^{+}, \mathrm{CD}^{+}$, and CD1d-restricted $\mathrm{T}$ cells. Similarly, HIV Nef thwarts not only MHC class I, MHC class II, CD4, and CD28, but also CD1a (18). It is remarkable that these viral proteins can act on molecules with such different primary structures. Moreover, it is somewhat surprising, but telling, that herpesviruses such as HCMV, with over 200 genes, target multiple recognition pathways using a single viral protein. This targeting is not incidental and further emphasizes the importance of hindering immune recognition.

\section{A model for CD1-restricted $T$ cell recognition of herpesvirus- infected cells}

KSHV and HIV reduce the cell-surface expression of CD1 molecules. We predict that other viruses, especially herpesviruses, will also be found to block expression of or antigen presentation by CD1. Because CD1-restricted T cells play a role in antiviral immunity, interrupting lines of communication with these $T$ cells is advantageous to the pathogen. If viruses do not alter cellular lipids, how does CD1 signal the presence of viruses? One answer may derive from the consideration of how CD1 molecules traffic in cells. Presentation of lipids by CD1 molecules requires traffic into endosomal compartments (6). CD1 molecules that cannot be internalized because they lack cytoplasmic domains or are expressed in cells without certain clathrin adaptors cannot present lipids to T cells (6). Thus, in our model depicted in Figure 1A, the immunologically relevant lipids recognized by CD1-restricted T cells are loaded onto CD1 in endosomes or lysosomes. Other lipids, e.g. phosphatidylinositol, are loaded onto nascent CD1 molecules in the ER and are less antigenic. Moreover, CD1 molecules are rapidly endocytosed from plasma membranes, and the bulk of these molecules amass in endosomal or lysosomal vesicles, not on the cell surface (6).

Structural proteins from herpesviruses, including HSV, VZV, and HCMV, extensively accumulate in the trans-Golgi network (TGN) and endosomes (19). Virus particles are assembled on the surfaces of the TGN and endosomes, causing enveloped virions 
to bud into cytoplasmic vesicles and later be transported to the cell surface (Figure 1B). At intermediate-to-late stages of HSV replication, both cellular and viral TGN/ endosomal proteins are redistributed to the cell surface (20). This redistribution is apparently produced through the action of HSV proteins that disrupt traffic so that endosomal proteins move to the cell surface, taking virus particles along for the ride. This egress pathway appears to be a basic process of all herpesviruses. We propose that substantial quantities of CD1 are relocalized from endosomes to the cell surface after herpesvirus infection (Figure 1B). By this model, CD1-bearing endosomal lipids emerge in substantial quantities on the cell surface and signal that viruses are present and have disrupted endosomal traffic. Redistribution of CD1 molecules from endosomes to the cell surface could be a general feature of other viral infections. Poxviruses and HIV can also assemble in endosomes $(19,21)$. This model of CD1 as a molecular flag is analogous to the role of MIC-A and MIC-B proteins, which signal to $\mathrm{T}$ and $\mathrm{NK}$ cells upon viral infection (1). However, as with all immune recognition events, herpesviruses counter with viral inhibitors that promote invisibility, at least for a time.

Address correspondence to: David C. Johnson, Department of Molecular Microbi- ology and Immunology, Oregon Health and Science University, 3181 South West Sam Jackson Park Road, Portland, Oregon 97239-3098, USA. Phone: (503) 494-0834; Fax: (503) 494-6862; E-mail: johnsoda@ohsu.edu.

1. Groh, V., et al. 2001. Costimulation of CD8alphabeta T cells by NKG2D via engagement by MIC induced on virus-infected cells. Nat. Immunol. 2:255-260.

2. Ploegh, H.L. 1998. Viral strategies of immune evasion. Science. 280:248-253.

3. Yewdell, J.W., and Hill, A.B. 2002. Viral interference with antigen presentation. Nat. Immunol. 3:1019-1025.

4. Hegde, N.R., Chevalier, M.S., and Johnson, D.C. 2003. Viral inhibition of MHC class II antigen presentation. Trends Immunol. 24:278-285.

5. Dunn, C., et al. 2003. Human cytomegalovirus glycoprotein UL16 causes intracellular sequestration of NKG2D ligands, protecting against natural killer cell cytotoxicity. J. Exp. Med. 197:1427-1439.

6. Brigl, M., and Brenner, M.B. 2004. CD1: antigen presentation and $\mathrm{T}$ cell function. Annu. Rev. Immunol. 22:817-890.

7. Godfrey, D.I., and Kronenberg, M. 2004. Going both ways: immune regulation via CD1d-dependent NKT cells. J. Clin. Invest. 114:1379-1388. doi:10.1172/JCI200423594.

8. Skold, M., and Behar, S.M. 2003. Role of CD1drestricted NKT cells in microbial immunity. Infect. Immun. 71:5447-5455.

9. van Dommelen, S.L., and Degli-Esposti, M.A. 2004. NKT cells and viral immunity. Immunol. Cell Biol. 82:332-341.

10. van Dommelen, S.L., Tabarias, H.A., Smyth, M.J., and Degli-Esposti, M.A. 2003. Activation of natural killer (NK) T cells during murine cytomegalovirus infection enhances the antiviral response mediated by NK cells. J. Virol. 77:1877-1884.

11. Grubor-Bauk, B., Simmons, A., Mayrhofer, G., and
Speck, P.G. 2003. Impaired clearance of herpes simplex virus type 1 from mice lacking CD1d or NKT cells expressing the semivariant $\mathrm{V}$ alpha 14-J alpha 281 TCR. J. Immunol. 170:1430-1434.

12. Ashkar, A.A., and Rosenthal, K.L. 2003. Interleukin-15 and natural killer and NKT cells play a critical role in innate protection against genital herpes simplex virus type 2 infection. J. Virol. 77:10168-10171.

13. Levy, O., et al. 2003. Disseminated varicella infection due to the vaccine strain of varicella-zoster virus, in a patient with a novel deficiency in natural killer T cells. J. Infect. Dis. 188:948-953.

14. Brigl, M., Bry, L., Kent, S.C., Gumperz, J.E., and Brenner, M.B. 2003. Mechanism of CD1d-restricted natural killer $\mathrm{T}$ cell activation during microbial infection. Nat. Immunol. 4:1230-1237.

15. Sanchez, D.J., Gumperz, J.E., and Ganem, D. 2005. Regulation of CD1d expression and function by a herpesvirus infection. J. Clin. Invest. 115:1369-1378. doi:10.1172/JCI200524041.

16. Coscoy, L., and Ganem, D. 2000. Kaposi's sarcomaassociated herpesvirus encodes two proteins that block cell surface display of MHC class I chains by enhancing their endocytosis. Proc. Natl. Acad. Sci. U. S. A. 97:8051-8056.

17. Coscoy, L., and Ganem, D. 2001. A viral protein that selectively downregulates ICAM-1 and B7-2 and modulates T cell costimulation. J. Clin. Invest. 107:1599-1606.

18. Shinya, E., et al. 2004. Endogenously expressed HIV-1 nef down-regulates antigen-presenting molecules, not only class I MHC but also CD1a, in immature dendritic cells. Virology. 326:79-89.

19. Johnson, D.C., and Huber, M.T. 2002. Directed egress of animal viruses promotes cell-to-cell spread. J. Virol. 76:1-8.

20. Wisner, T.W., and Johnson, D.C. 2004. Redistribution of cellular and herpes simplex virus proteins from the trans-golgi network to cell junctions without enveloped capsids. J. Virol. 78:11519-11535.

21. Pelchen-Matthews, A., Kramer, B., and Marsh, M. 2003. Infectious HIV-1 assembles in late endosomes in primary macrophages. J. Cell Biol. 162:443-455. 